\title{
Ischaemic Stroke Complicating Infective Endocarditis: Microbleeds Are the Diagnostic Clue
}

\author{
Wadi Bnouhanna, Taho Oulounao Adlaide, Chaima EL Jemili, Mounia Rahamani, \\ Maria Benabdeljlil, Saadia Aidi
}

Department of Neurology A and Neuropsychology, Hospital of Specialties, Ibn Sina University Hospital, Faculty of Medicine and Pharmacy of Rabat, Mohammed V University, Rabat, Morocco

Email: w.bnouhanna@gmail.com, ouniarahmani4@gmail.com, benab.maria@yahoo.fr, aidi_sadia@yahoo.fr

How to cite this paper: Bnouhanna, W., Adlaide, T.O., EL Jemili, C., Rahamani, M., Benabdeljili, M. and Aidi, S. (2022) Ischaemic Stroke Complicating Infective Endocarditis: Microbleeds Are the Diagnostic Clue. Case Reports in Clinical Medicine, 11, 13-18. https://doi.org/10.4236/crcm.2022.111003

Received: December 4, 2021

Accepted: January 16, 2022

Published: January 19, 2022

Copyright ( 2022 by author(s) and Scientific Research Publishing Inc. This work is licensed under the Creative Commons Attribution International License (CC BY 4.0).

http://creativecommons.org/licenses/by/4.0/

\begin{abstract}
Introduction: Ischemic strokes represent a classic complication of infective endocarditis (IE) and are most often related to the fragmentation of valvular vegetation. In most cases, they occur in the Sylvian territory and are related to Staphylococcus aureus. MRI can demonstrate, in addition to the AVCI image, cerebral microbleeds (CMBs) that are very suggestive of the diagnosis. We present the case of a patient who presented an ischemic stroke occurring in the context of infective endocarditis. Observation: A 32-year-old woman with no previous medical history initially presented with acute febrile headache, two weeks later she developed right hemiplegia with aphasia in the context of fever. Her general examination revealed lesions on the soles of her feet, in the form of nodules, infected in places, suggestive of Janeway nodules (characteristic of IE). The brain MRI showed an ischemic stroke in the Sylvian territory, with the presence of multiple microbleeds on the magnetic susceptibility sequences. The biological examination showed a severe inflammatory syndrome; the transthoracic echocardiography (TTE) confirmed the presence of vegetation on the aortic valve; and the blood cultures showed a Staphylococcus aureus. Antibiotic therapy was started, and the patient was transferred to the cardiology department. Discussion: Our observation has a double interest. The first is clinical due to the presence of plantar nodules (Janeway's nodules) that are characteristic of IE which should orientate the diagnosis before the installation of the stroke, and the second is radiological and linked to the discovery of microbleeds. Their presence is increasingly reported in the literature, but their pathophysiology is not yet very clear. Conclusion: Stroke is the most frequent extracardiac complication during IE. The presence of microbleeds contributes to early diagnosis, especially in asymptomatic forms.
\end{abstract}




\section{Keywords}

Ischaemic Stroke, Infective Endocarditis, Brain MRI, Microbleeds

\section{Introduction}

Infective endocarditis (IE) is a bacterial attack on the endocardium, responsible for valvular lesions, most frequently the mitral valve (43.3\%), followed by the aortic valve (26.3\%) with a significant risk of heart failure and septic embolism.

Staphylococci are currently the most frequently isolated organisms in IE. The diagnosis is based on a combination of clinical, microbiological and echocardiographic evidence.

Janeway lesions are haemorrhagic, irregular and non-painful palmoplantar macules. They are seen with the acute form of bacterial endocarditis and are part of the modified DUKES minor criteria [1].

Ischaemic strokes are the most common neurological complications. They are most often manifested by a sudden focal neurological deficit (hemiparesis, hemianesthesia, hemianopsia, etc.), permanent or transient, and occurring in a febrile context. However, some of them may be completely asymptomatic and detected only on the occasion of a systematic imaging examination.

\section{Case Presentation}

A 32-year-old woman, a mother of 4 children and without any particular history, presented on 09 August 2021 with a fever of 40, headaches and vomiting, and the appearance of very painful skin lesions in the form of nodules located on the soles of the feet, some of which are purplish in color (Figure 1: photos taken by the husband), characteristic of Janeway nodules.

On 21 September 2021, the patient suddenly developed weakness of the right hemicbody with the suspension of speech, in the context of fever. The general examination results are fever $40, \mathrm{BP}=140 / 07$, tachycardia 120 , and polypnoea $30 \mathrm{cycles} / \mathrm{min}$. The neurological examination found a conscious patient with total right hemiplegia 0/5, global aphasia, and an NHISS score of 14 . The cardiovascular examination showed a heart murmur in all 4 foci. Finally, the dermatological examination noted scarring lesions with significant desquamation on the soles of the feet.

Brain MRI without and with gadolinium (21 September 2021) was in favor of ischemic stroke (Figure 2) associated with an occlusion of the intracranial left internal carotid artery (Figure 3) and multiple bi-hemispheric cortical and sub tentorial microbleeds (Figure 4). The echocardiography showed mobile elements in the aortic valve of $10 \mathrm{~mm}$ and $6 \mathrm{~mm}$ in favor of infective endocarditis on aortic valve disease. Blood culture returned positive with isolation of Staphylococcus aureus. The cerebrospinal fluid (CSF) analysis was in favour of bacterial meningitis with positive culture for Staphylococcus aureus (210 GB (40\% PNN, 
$60 \%$ lymphocytes) and Proteinorachy at $0.33 \mathrm{~g} / \mathrm{l}$, glycorrhaphy at $0.67 \mathrm{~g} / \mathrm{l}$ ). The patient was put on Ampicillin $12 \mathrm{~g} /$ day associated with gentamycin $160 \mathrm{mg} /$ day for 6 weeks. The patient continued to improve neurologically with the beginning of recovery of the motor deficit, on the cardiological aspect a surgical intervention is planned.

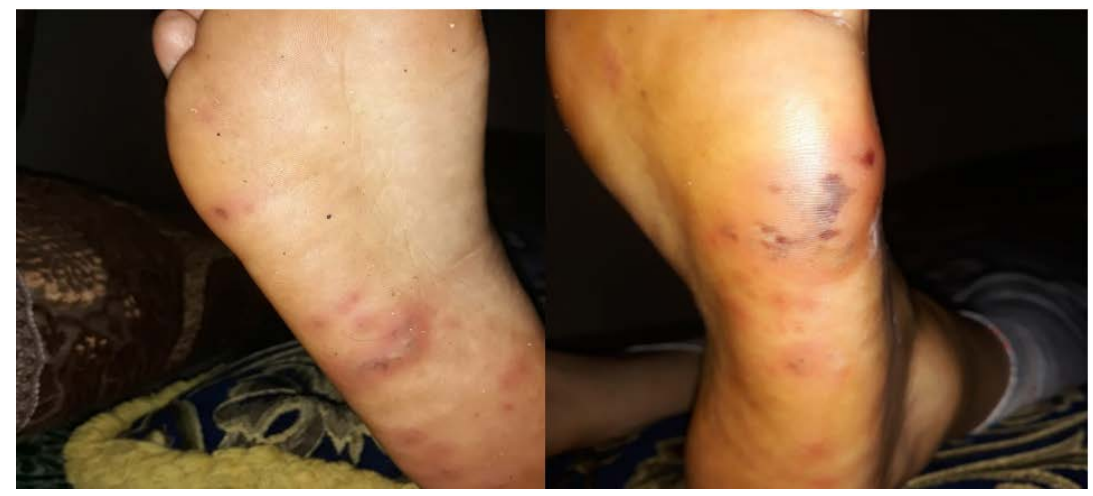

Figure 1. Janeway plantar nodules.

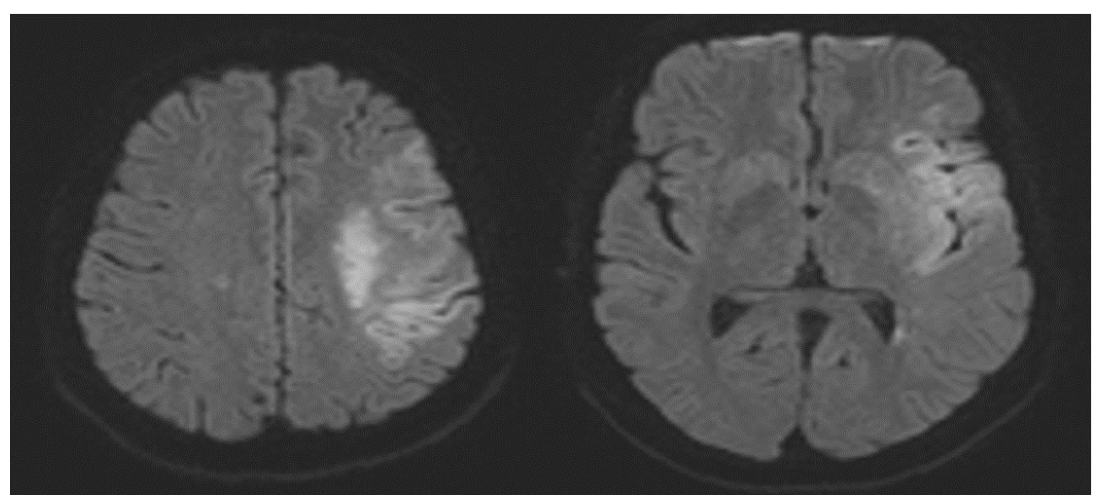

Figure 2. Axial DWI brain MRI: Hypersignal DWI in the fronto-parieto-insular regions corresponding to ischemic stroke of the superficial and deep MCA territory.
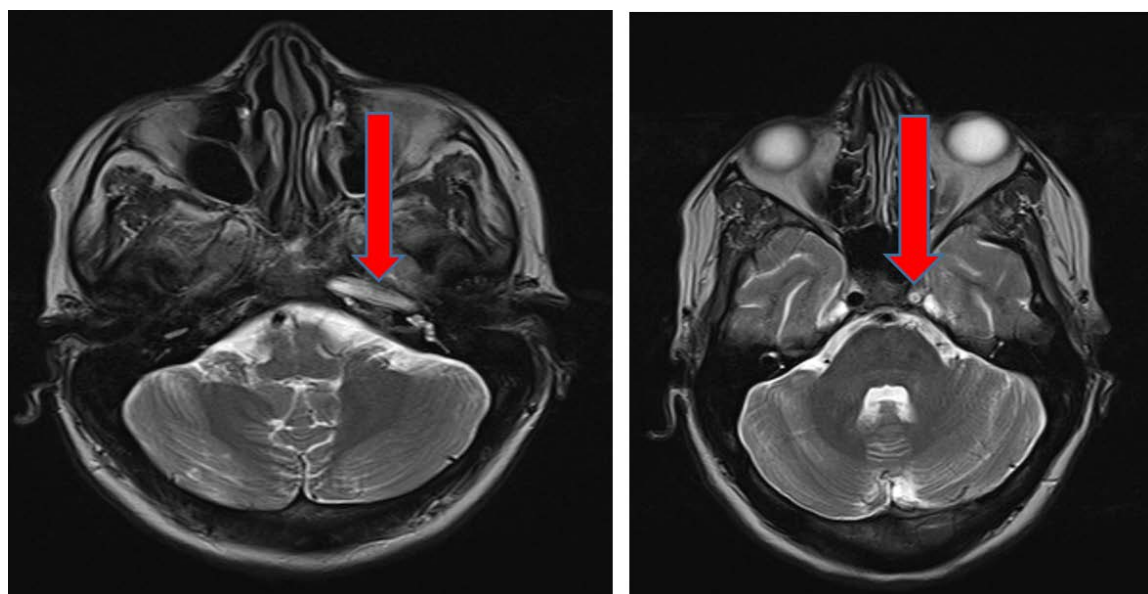

Figure 3. T2 axial brain MRI: T2-Falair hypersignal of the left internal carotid artery in its intra petrous segment (red arrow), in favour of an occlusion of the left intracranial internal carotid artery. 




Figure 4. SWI axial brain MRI: Multiple microbleeds in the cortico-subcortical and subtentorial areas (cerebellum and brainstem).

\section{Discussion}

Neurological manifestations are the most frequent extracardiac complication in IE. They are most often of cerebrovascular origin, resulting from the migration of a fragment of vegetation (ischaemia) or the rupture of a weakened arterial wall (haemorrhage). They are asymptomatic in $30 \%$ to $50 \%$ of cases. Symptomatic stroke is the second most common cause of death from IE [2].

The incidence of embolic events in IE is estimated to be between $10 \%-50 \%$ [3]. In addition, ischaemic stroke accounts for approximately $70 \%$ of cerebrovascular events in patients with bacterial endocarditis who have a stroke. Risk factors for cerebral embolisation include $S$. aureus endocarditis, large vegetation, increased mobility of vegetation, and mitral valve involvement [4]. Haemorrhagic strokes account for about $30 \%$ of cerebrovascular complications. They are due to haemorrhagic transformation of cerebral infarction, primary intracerebral haemorrhage, or ruptured cerebral mycotic aneurysm [5].

The frequency of microbleeds has been estimated at $57 \%$ in patients who had brain MRI within seven days of hospital admission for IE [6]. These microbleeds are preferentially cortical in distribution, but deep involvement is possible [7].

The pathophysiological process involved in the formation of these microhemorrhages is still to be clarified. The cortical location of microbleeds may reflect a preferential mode of entry of septic emboli across the blood-brain barrier at the cortico-pial junction. Microbleeds may also be due to a subacute process, either due to immunological vasculitis and/or an embolic process in the vasa vasorum [8].

The prognostic value of cerebral microbleeds has been assessed in several studies. All concluded that the presence and the number of CMBs, particularly two 
or more microbleeds, was an independent predictor of the development of intracerebral haemorrhage. Based on all these arguments, several authors propose to include microbleeds in the diagnostic criteria for infective endocarditis.

The performance of valve surgery in the acute phase of EI and the delay in the procedure pose a problem in the event of neurovascular complications. Indeed, there is a cerebral haemorrhagic risk directly related to the extracorporeal circulation, and to the curative anticoagulation necessary after the intervention. The inclusion of microbleeds may be useful to evaluate the risk of IE patients developing major postoperative cerebral bleeding [9].

\section{Conclusion}

Stroke is the most frequent extracardiac complication during IE. The presence of microbleeds is increasingly reported in studies and may contribute to early diagnosis, especially in asymptomatic forms, as does the clinical presence of nodular Janeway skin lesions. Brain MRI should be performed systematically in cases of bacterial IE, even in the absence of neurological signs, to detect asymptomatic lesions and guide the therapeutic strategy.

\section{Acknowledgements}

We thank our colleagues from the Neurology A and Neuropsychology department as well as the Neuroradiology team of the Hospital of Specialties, Ibn Sina University Hospital for their and expertise that greatly assisted our research.

\section{Conflicts of Interest}

The authors declare no conflicts of interest regarding the publication of this paper.

\section{References}

[1] Marie, T.J. (2008) Osler's Nodes and Janeway Lesions. The American Journal of Medicine, 121, 105-106. https://doi.org/10.1016/j.amjmed.2007.07.035

[2] Murdoch, D.R., Corey, G.R., Hoen, B., Miro, J.M., Fowler Jr., V.G., Bayer, A.S., et al. (2009) Clinical Presentation, Etiology, and Outcome of Infective Endocarditis in the 21st Century: The International Collaboration on Endocarditis-Prospective Cohort Study. Arch Internal Medicine, 169, 463-473. https://doi.org/10.1001/archinternmed.2008.603

[3] Cantier, M., et al. (2017) Neurologic Complications of Infective Endocarditis: Recent Findings. Current Infectious Disease Reports, 19, Article No. 41. https://doi.org/10.1007/s11908-017-0593-6

[4] Garcia-Cabrera, E., Fernandez-Hidalgo, N., Almirante, B., et al. (2013) Neurological Complications of Infective Endocarditis: Risk Factors, Outcome, and Impact of Cardiac Surgery: A Multicenter Observational Study. Circulation, 127, 2272-2284. https://doi.org/10.1161/CIRCULATIONAHA.112.000813

[5] Schirone, L., Iaccarino, A., Saade, W., D’Abramo, M., De Bellis, A., Frati, G., Sciarretta, S., Mestres, C.-A. and Greco, E. (2018) Cerebrovascular Complications and Infective Endocarditis: Impact of Available Evidence on Clinical Outcome. BioMed 
Research International, 2018, Article ID: 4109358, 6 p. https://doi.org/10.1155/2018/4109358

[6] Klein, I., Iung, B., Labreuche, J., et al. (2009) Cerebral Microbleeds Are Frequent in Infective Endocarditis: A Case Control Study. Stroke, 40, 3461-3465.

https://doi.org/10.1161/STROKEAHA.109.562546

[7] Sonneville, R., Mirabel, M., Hajage, D., et al. (2011) Neurologic Complications and Outcomes of Infective Endocarditis in Critically Ill Patients: The ENDOcardite in REAnimation Prospective Multicenter Study. Critical Care Medicine, 39, 1474-1481. https://doi.org/10.1097/CCM.0b013e3182120b41

[8] Grecu, N., Tiu, C., Terecoasa, E. and Bajenaru, O. (2014) Endocarditis and Stroke. Maedica (Bucur), 9, 375-381.

[9] Murai, R., Kaji, S., Kitai, T., Kim, K., Ota, M., Koyama, T. and Furukawa, Y. (2019) The Clinical Significance of Cerebral Microbleeds in Infective Endocarditis Patients. Seminars in Thoracic and Cardiovascular Surgery, 31, 51-58.

https://doi.org/10.1053/j.semtcvs.2018.09.020 